CAHIERS DE

NARRATOLOGIE

\section{Cahiers de Narratologie}

Analyse et théorie narratives

$38 \mid 2020$

Lusor in Fabula. Jeu vidéo et nouvelles frontières du récit

\title{
Improvisation et réécriture narrative : jouer au mode libre de GTA Online
}

Iñigo Atucha

\section{(2) OpenEdition \\ Journals}

Electronic version

URL: http://journals.openedition.org/narratologie/11687

DOI: 10.4000/narratologie.11687

ISSN: 1765-307X

Publisher

LIRCES

Electronic reference

Iñigo Atucha, "Improvisation et réécriture narrative : jouer au mode libre de GTA Online", Cahiers de Narratologie [Online], 38 | 2020, Online since 18 January 2021, connection on 29 January 2021. URL: http://journals.openedition.org/narratologie/11687 ; DOl: https://doi.org/10.4000/narratologie. 11687

This text was automatically generated on 29 January 2021.

Article L.111-1 du Code de la propriété intellectuelle. 


\title{
Improvisation et réécriture narrative : jouer au mode libre de GTA Online
}

\author{
Iñigo Atucha
}

\section{L'impératif d'action}

1 Je roule vite, sans doute trop vite, je dépasse par la droite la colonne des véhicules qui attendent au feu rouge. Un camion change subitement de direction et entre en collision avec ma voiture, qui fait plusieurs tonneaux. Ma voiture n'a plus de capot, il manque une portière, le pare-brise est en miettes, mais je continue ma route et, lorsque j'arrive au croisement suivant, un automobiliste me coupe la route et nos deux voitures finissent sur le toit, au milieu des piétons qui tentent de fuir.

2 Je monte à bord d'un taxi que je viens d'appeler et, pour rejoindre ma destination, je propose au conducteur un itinéraire qui passe par des sentiers périlleux au milieu des falaises. À chaque tentative, mon trajet finit invariablement au fond du ravin.

3 Je pénètre dans l'enceinte de la base militaire. Mon indice de recherche passe instantanément à quatre étoiles, les militaires surgissent de toute part et ouvrent le feu sur moi. Je survis comme je peux, ils sont trop nombreux, et je finis toujours par mourir misérablement.

4 Ces descriptions, qui sont autant de bribes de récits, racontent l'irruption d'un événement imprévu, mais dont certains contours ont parfois fait l'objet d'une préparation, qui se produit durant certaines phases de jeu du mode libre (freeroam) de GTA Online. Ces phases sont à la croisée de la flânerie, de l'ennui, de l'improvisation et parfois du jeu collectif qui comporte un ensemble très réduit de règles élémentaires. Elles interrogent « l'impératif d'action » dans GTA Online, structuré par une mécanique du jeu qui s'articule autour de fragments de récits déposés dans le jeu. L'idée est ici d'esquisser quelques idées concernant la circulation des récits et des niveaux de récit et la mise en action des fragments de narration placés dans le jeu. Les objets et les 
accessoires mis à disposition dans le jeu tiennent un rôle central : revêtir certains vêtements, se servir de certaines armes, acheter une voiture sont autant de façons de prendre possession d'un fragment d'histoire, de représenter une part d'un récit cinématographique, télévisuel ou littéraire, sous une forme nouvelle et hybride. Le plus souvent, en rejouant certaines scènes de façon imparfaite et improvisée, dans un esprit opportuniste et syncrétique qui finit par rendre méconnaissables les éléments repris des différents corpus culturels. Les pratiques d'improvisation seront également au centre de cette contribution, dans la mesure où elles permettent de suggérer une compréhension du mode Online qui passe par un rapport d'analogie avec certaines pratiques musicales ou théâtrales.

5 Mais GTA Online, en tant que terrain de jeu, présente un intérêt supplémentaire : en effet, une partie des fragments de récits provient de la narration prescrite dans le mode histoire (storymode) de GTA V, un récit que les concepteurs ont déposé dans le jeu et que les joueurs actualisent en suivant différents embranchements. Le récit qui naît ainsi des actions des joueurs retrace un parcours particulier, tout en reposant sur les éléments de narrativisation présentés par l'univers virtuel, qui orientent l'action et qui ont fait naître une histoire à partir des actions requises auprès des joueurs ${ }^{1}$. GTA Online, en tant que versant multijoueur du monde de GTA V, se place donc à la croisée de cette superposition de récits, tout en se présentant comme un recyclage indépendant du mode histoire, dont elle reprend un grand nombre d'éléments et un certain nombre de personnages. Ce recyclage a été prolongé et reconfiguré dès l'origine par l'exploitation commerciale du mode Online, qui s'est ensuite avérée bien plus longue que celle du mode histoire, avec des contenus additionnels encore implémentés aujourd'hui.

\section{GTA Online}

6 GTA Online est un monde ouvert persistant, accessible en multijoueur. Il est mis à la disposition des joueurs le 1er octobre 2013. Il permet l'accès à des sessions (lobby), auxquelles 16 ou 30 joueurs $^{2}$ peuvent se connecter simultanément. GTA Online est accessible gratuitement pour tous les joueurs ayant acquis GTA V qui, au moment de sa commercialisation en septembre 2013, est la nouvelle version très attendue de la franchise Grand Theft Auto. Éditée par le studio Rockstar, celle-ci existe depuis 1997. Si les premières versions proposent un jeu dans un monde ouvert, mais en deux dimensions et en vue aérienne pixelisée, la représentation en trois dimensions avec vue à la troisième personne apparaît dans GTA III, commercialisé en $2001^{3}$. Reprenant les mêmes principes et des graphismes similaires, GTA Vice City paraît en 2002, puis GTA San Andreas en 2004. Il faut compter quatre années supplémentaires pour voir paraître GTA IV, en 2008-2009 (en fonction des plateformes), qui marque un tournant en raison de la grandeur de la map proposée et du souci des détails.

7 L'action de GTA V se déroule sur l'île de San Andreas. Contrairement aux autres versions du jeu, GTA V propose non seulement un mode histoire, mais également un mode multijoueur (baptisé donc GTA Online) qui utilise le même décor. Cependant, l'exploitation commerciale et l'immense succès rencontré par le mode Online qui, 7 ans après sa mise en ligne, connaît toujours une fréquentation importante, a modifié la promesse initiale du monde ouvert multijoueur et l'économie propre à ce mode : les conséquences de cette longue exploitation se sont fait sentir sur les contenus 
additionnels régulièrement implémentés dans le jeu et sur l'impact qu'ils ont eu sur l'expérience des joueurs.

\section{GTA V} réplique insulaire d'une Californie dystopique qui se retrouverait au milieu de l'océan pacifique, dans la droite ligne de la satire critique de la société américaine qui caractérise la série GTA${ }^{4}$. La ville principale de San Andreas est Los Santos, réplique caricaturale et dysfonctionnelle de la ville de Los Angeles.

9 Le joueur incarne (et c'est là l'originalité de cette version du jeu), trois personnages, Franklin, Michael et Trevor. Ces trajectoires narratives distinctes permettent une démultiplication des points de vue sur Los Santos. Les trois récits sont tout d'abord dispersés, pour ensuite se réunir très progressivement en une trame unique. Franklin vit chez sa tante, du côté des quartiers de Strawberry et de Chamberlain Hills, deux quartiers très populaires de Los Santos. Michael est un ancien braqueur qui coule des jours heureux dans les abords des beaux quartiers. Et Trevor, ancien complice de Michael, a trouvé refuge dans Blain County, l'arrière-pays de San Andreas, désertique et sauvage. Une chaîne de hautes collines sépare Los Santos de Blaine County, alors que le mont Chilliad, situé à l'extrême nord de la map, surplombe l'ensemble de l'île.

Chaque personnage inscrit sa trajectoire individuelle dans la trame principale de l'histoire. La division en trois points de vue est prolongée par un découpage spatial de San Andreas qui répond au statut social des trois personnages. La trajectoire (dans un sens très concret) de Franklin est la plus remarquable : de la petite maison de sa tante, où il occupe une chambre, il accède rapidement à une villa moderne et luxueuse sur les hauteurs de Los Santos, qu'il ne possède pas, mais où il peut séjourner indéfiniment ${ }^{5}$. Sa trajectoire spatiale renvoie directement à sa trajectoire sociale, celle d'une ascension qui passe par toutes sortes d'activités criminelles. Trevor réalise quant à lui un chemin circulaire en deux temps : après avoir retrouvé la trace de Michael qu'il croyait mort, il passe de sa caravane délabrée située à Sandy shores à un appartement de Los Santos, qu'il occupe en menaçant son propriétaire, pour ensuite retrouver son mobile home initial. Michael est le seul des trois protagonistes à ne pas changer de domicile : à aucun moment il ne quitte sa villa luxueuse, qui symbolise une réussite ostentatoire, mais que l'on devine fragile, puisqu'elle est le fruit de ses activités criminelles.

11 Le récit du mode histoire fait ainsi écho à de nombreuses sources, tant littéraires que cinématographiques ${ }^{6}$ : on retrouve des accents qui pourraient faire penser entre autres à la trilogie Underworld USA de James Ellroy ${ }^{7}$, ainsi qu'au film Scarface ${ }^{8}$ et plus encore au film Heat ${ }^{9}$, sans oublier Reservoir Dogs ${ }^{10}$. Des missions du jeu renvoient directement à des séquences de films, par exemple la mission «Conseil conjugal» («Marriage Counseling ", mission réalisée par Michael et Franklin, qui doivent arracher les colonnes de soutien d'une villa pour que celle-ci s'effondre) qui reprend une scène de l'Arme fatale $2^{11}$, les consultations de Michael auprès du psychologue font évidemment penser à celle de Paul Vitti, personnage de parrain mafieux joué par Robert De Niro dans Mafia Blues ${ }^{12}$.

12 Ce réseau d'influences varié donne au récit des résonances multiples et San Andreas, en tant que décor, donne ainsi corps à des trajectoires narratives et sociales complexes et s'inscrit dans un cadre dystopique : le cadre des valeurs est renversé, les joueurs, qui 
incarnent trois délinquants, tentent d'échapper à la police, organisent des braquages, volent des voitures, assassinent contre de l'argent, organisent un réseau de trafic de voitures, et ainsi de suite.

13 La vie à l'intérieur de ce cadre dystopique, que ce soit dans le mode histoire ou dans le mode libre, est rythmé par un régime de temporalité défini de façon à la fois imprécise et circulaire. Le cycle circadien est totalement détaché de toute référence aux saisons ou aux années ${ }^{13}$. Les journées et les nuits se succèdent à Los Santos, sans marquer cependant aucune progression du calendrier. Mais cette alternance a lieu en suivant un rythme accéléré : une heure dans le jeu équivaut à deux minutes dans la vie réelle. C'est donc une sorte de jour sans fin, sans date, sans saison, uniquement marqué par les variations météorologiques et la succession rapide des heures. Los Santos est ainsi un décor dont la conception mimétique ${ }^{14}$ s'arrête aux éléments matériels et météorologiques, à peine marqué par l'alternance du jour et de la nuit. C'est une toile de fond naturaliste qui fait illusion, qui contient le lieu scénique dans un espace à la temporalité circulaire.

Cependant, le joueur du mode Online ne vit pas exactement dans le monde du mode histoire, même si le récit du mode Online s'inscrit dans le même espace géographique et que certains personnages du mode histoire jouent aussi un rôle dans le mode Online $\mathrm{e}^{15}$. Il existe des différences entre les deux versions de ce monde : pour des raisons techniques, les animaux ont été exclus du mode multijoueur, ainsi que les avions de ligne qui, dans le mode histoire, sillonnent dans le ciel ou qui stationnent sur le tarmac à l'aéroport de Los Santos. Le nombre de piétons est inférieur et la variété de la population est moindre. De même en ce qui concerne le nombre et le style des véhicules en circulation dans les rues, qui diffèrent de manière sensible.

Le récit déposé dans le mode histoire se retrouve ainsi en partie dans le mode Online. La continuité narrative et spatiale permet de passer de l'un à l'autre en ne provoquant qu'un dépaysement léger.

\section{Joueur au mode libre}

16 Au-delà des interdépendances entre les deux modes, un élément important, qui n'était sans doute pas prévu (en tout cas pas dans cette ampleur) lors de la commercialisation $\mathrm{du}$ jeu, est venu alimenter l'indépendance progressive du mode online par rapport au mode histoire. En raison de l'immense succès de GTA Online, l'exploitation commerciale du jeu s'est concentrée de manière quasiment exclusive sur le développement de contenus additionnels pour le mode en ligne et, cela, de novembre 2013 à août $2020^{16}$. L'extension du mode libre a donc impliqué une évolution des schémas narratifs proposés: l'horizon du jeu s'étend aujourd'hui bien au-delà des braquages et d'un Los Santos de film d'action policier, il comporte un vaste arsenal militaire, un canon orbital, des voitures volantes, des avions en tous genres, des accessoires futuristes, des bases souterraines, des boîtes de nuit, etc.

Étant donné cet environnement, «jouer » à GTA Online peut recouvrir une multitude comportements différents à l'intérieur de l'espace mis à disposition. Une multitude de missions sont proposées. Ce genre d'activité se rapproche de celles qui sont proposées dans le mode histoire, à la différence que le mode Online permet, voire exige, de les réaliser avec d'autres joueurs. La dimension coopérative du jeu en ligne est ici fondamentale. D'autres formes de jeux collectifs (courses, modes adversaires, missions 
de contact, etc.) sont accessibles directement à travers un système de menus, d'invitations et de sessions privées, et ils ont été eux aussi très largement alimentés au cours de ces dernières années. Cependant, certaines activités ont lieu au sein même de la session principale ${ }^{17}$. Leur déroulement au milieu des autres joueurs est bien entendu l'objet d'une mise en tension délibérée: il s'agit par exemple de courir le risque de perdre une précieuse cargaison en raison de l'agressivité arbitraire d'autres joueurs, toujours susceptibles de semer la destruction par pur amusement.

\section{Mesurer la progression}

18 La progression dans le mode histoire n'est mesurable que par le pourcentage de réalisation indiqué lors de la sauvegarde de la partie. Pour le reste, il n'y a que quelques indicateurs de compétence (discrétion, pilotage, endurance, etc.) et l'argent accumulé sur le compte en banque pour orienter l'avancée du joueur ${ }^{18}$.

19 Le mode Online encourage quant à lui l'activité du joueur, à travers la progression des niveaux, basée sur une échelle ouverte, ainsi qu'à travers une accumulation d'argents et d'objets. Le niveau (level) du joueur augmente au fur et à mesure de la réalisation de missions, de courses, d'acquisition de ressources, etc. Mais il donne accès à bien peu de choses: à peine débloque-t-il, à partir du niveau 100, tous les accessoires de customisation destinés à personnaliser et améliorer les performances des voitures; et, à partir du niveau 120, le joueur peut acquérir toutes les armes en vente dans le jeu. Ainsi, dans le mode libre, le level est avant tout un marqueur de compétence : il indique un niveau d'expérience, une connaissance générale du jeu qui peut décourager les agressions hasardeuses et gratuites de la part d'autres joueurs ou, au contraire, les encourager à demander de l'aide.

20 Comme dans le mode libre, la progression se mesure avant tout à travers l'accumulation d'argent: l'argent permet de se procurer des accessoires et des véhicules, il permet en particulier l'acquisition d'appartements, de boîtes de nuit, de bunkers, des hangars militaires et des quartiers généraux souterrains qui donnent accès à de nouvelles missions et étendent la vie du jeu de façon importante.

21 L'abondance matérielle et l'accumulation d'argent sont deux ressorts importants de GTA Online. Couplés à l'importance symbolique et sociale accordée au niveau, ils alimentent la motivation du joueur à venir et revenir dans le jeu afin d'augmenter son patrimoine, cette dynamique étant encouragée par Rockstar qui a multiplié les contenus additionnels, dont la valeur répond à un taux d'inflation toujours plus important. Le joueur, expérimenté ou novice, passe donc son temps à dépenser l'argent qu'il vient à peine de gagner, en passant des heures à collecter des ressources et à faire fonctionner ses affaires commerciales (illicites, la plupart du temps), et ainsi de suite en suivant un cycle sans fin.

\section{Improvisations}

Mais, au-delà des activités aménagées et balisées dans le jeu libre ou dans les sessions dédiées aux activités lucratives, que se passe-t-il dans les moments de creux? Qu'est-ce qui est en jeu dans ces moments d'ennuis, où l'on s'approche des autres joueurs et 
qu'une activité se met spontanément en place, en tant qu'extension ludique et spontanée de cette première rencontre?

Les quelques exemples livrés précédemment, à savoir l'attaque de la base militaire, le trajet hasardeux en taxi, les accidents de voiture plus ou moins délibérés, donnent une idée de ces activités qui ne répondent à aucune visée particulière concernant la progression dans le jeu, mais qui relèvent plus de l'improvisation, de l'exercice à peine organisé, de l'expérimentation avec le jeu ${ }^{19}$.

Il y a deux aspects de l'improvisation qui peuvent être à l'œuvre dans ces situations : la première est celle, liée à la flânerie, qui s'apparente à un vagabondage sans but particulier à travers la map, une libre déambulation uniquement guidée par une stratégie opportuniste qui se contente d'arpenter le territoire et de voir ce qui peut surgir, aussi bien en termes de rencontres que d'événements imprévus. Il est sans doute moins question, dans ces cas, d'appliquer une grille de lecture issue de l'improvisation au jazz, telle qu'elle sera présentée ci-dessous. Sans doute y a-t-il un air de famille avec quelque chose qui pourrait s'apparenter à de l'improvisation théâtrale, un jeu spontané (pour ne pas dire inconscient) avec des syntagmes symboliques issus de la culture cinématographique ou littéraire, déposés dans les objets et les accessoires proposés par GTA Online.

Un autre aspect de l'improvisation est bien plus proche de l'improvisation au jazz ou à celle en œuvre dans le spectacle vivant. Elle correspond à une élaboration de plus en plus structurée des activités spontanées, qui répondent à la question «à quoi on joue maintenant? » en y intégrant un degré d'organisation variable et, bien souvent, un nombre croissant de joueurs. Il s'agit au fond de suggérer que l'activité libre, mais organisée, qui joue avec le jeu, puise sa source dans l'événement impromptu et dont on ne cherche pas la survenue, mais dont on cherche à répéter l'expérience une fois qu'il est arrivé, en modifiant légèrement les conditions de réalisation.

Ainsi, un accident de la circulation qui se produit de façon brutale et inattendue peut sembler effrayant, agaçant, voire amusant, et le joueur cherche à en reproduire les manifestations en perfectionnant les conditions de sa survenue. Au premier accident suivent d'autres accidents, bien moins fortuits, provoqués par le joueur qui joue avec les mécaniques du jeu (les mécanismes de la circulation routière dans GTA, dans ce cas précis), pour en améliorer les qualités esthétiques. Et donc narrative («c'était un beau choc frontal », «j'ai détruit ma voiture après trois tonneaux »). Et cela est d'autant plus marquant que le trafic dans GTA Online semble être conçu pour que les véhicules conduits par des personnages non jouables entrent en collision avec les joueurs.

27 À l'imprévu succède la répétition, qui précise les contours de l'action selon des critères esthétiques et pratiques. Au récit d'un imprévu succède le récit d'une série d'actions coordonnées afin de provoquer une situation ou un événement.

Dans ce cadre précis, celui défini par GTA Online en tant que plateforme ludique obéissant à un certain nombre de règles et supposant un certain nombre de caractéristiques (qui définissent, ensemble, ce qu'il est possible de faire ou impossible de faire dans l'espace du jeu), il s'agit donc de produire des situations qui laissent une grande part à l'imprévu, aussi bien en ce qui concerne les probabilités de leur survenue qu'en ce qui concerne leur déroulement. Et qui, à partir de cet imprévu, admettent ensuite un certain degré d'organisation. Pour enfin aboutir au récit de la survenance de 
ces situations, de leurs variations (parfois infinitésimales) et des imprévus qui ont surgi en chemin.

\section{Qu'est-ce qu'on joue, maintenant ?}

L'étude que les sociologues Howard Becker et Robert Faulkner ont consacrée aux pratiques d'improvisation dans le jazz peut fournir quelques indications utiles à cet égard. Becker et Faulkner s'appuient sur leur longue pratique en tant que joueurs de jazz (ils sont des habitués des bars et des clubs dans lesquels ils jouent régulièrement et se qualifient eux-mêmes de musiciens semi-professionnels). Leur étude, qui s'intitule " Qu'est-ce qu'on joue, maintenant?», Le répertoire de jazz en action ${ }^{20}$ propose une analyse concernant la façon qu'ont les joueurs de jazz de se coordonner en vue d'improviser les morceaux qu'ils jouent ensemble dans les clubs et les bars. À propos de ce processus d'improvisation, Becker et Faulkner proposent la description suivante :

Si l'on voulait en donner un résumé, forcément (trop) court, on pourrait dire qu'un jazzman joue de manière banale et tout à fait routinière des versions de morceaux qu'il connaît déjà - ou dont il devine la structure - en substituant à la mélodie originale des mélodies composées dans le feu de l'action [...]. L'improvisation en jazz est (plus ou moins) un mélange de spontanéité et de conformité vis-à-vis d'une sorte de format préétabli ${ }^{21}$.

Ils proposent de distinguer le répertoire individuel, c'est-à-dire les morceaux que connaît le musicien et qui forment un réservoir de titres appris au cours des années, et le répertoire de travail, c'est-à-dire le répertoire commun, que les musiciens qui jouent ensemble peuvent exploiter. Ce répertoire est le fruit de négociations, de propositions et de réflexions, qui s'effectuent en quelques secondes, alors que les musiciens sont parfois déjà sur scène.

Les musiciens, en tant qu'individus qui interprètent les morceaux, sont les sujets d'un apprentissage et d'une connaissance, parfois imparfaite et partielle, dont ils doivent négocier la mise en action dans le cadre de situations concrètes. Ces situations peuvent être de nature commerciale ou privée, elles définissent en tous les cas la demande sur ce que l'on peut jouer, ce que l'on doit jouer et ce qu'il ne faut pas jouer.

Becker et Faulkner insistent sur la dimension interactive de l'improvisation, qui confronte le répertoire individuel de chaque musicien (à savoir l'ensemble des thèmes appris au cours des années d'activité et d'expériences, qui va de la connaissance vague d'un titre à une connaissance profonde, née d'une pratique intensive), au répertoire de travail (c'est-à-dire le répertoire commun proposé et négocié, en quelques secondes parfois, à partir des différents répertoires individuels en présence).

Dans le cas de GTA Online, ce qui est en jeu est un ensemble de répertoires culturels et ludiques dont les joueurs s'approprient et qui permettent la mise en œuvre d'un ensemble de pratiques et d'actions concrètes dans le jeu. Le joueur est ainsi celui qui agit dans l'espace scénique en interprétant un personnage, mais sans prononcer un seul mot, munis d'un canevas comportemental plus ou moins défini qu'il s'est souvent bricolé lui-même, à l'aide des accessoires vestimentaires, des armes et des véhicules dont il fait usage dans le cadre défini d'une session du mode libre.

Pour pouvoir être mis en œuvre, ce jeu improvisé propre au joueur demande la mise en mouvement d'un corps, au milieu d'un décor et muni d'un certain nombre d'accessoires. 


\section{Mise en action de la culture}

casque et dun mo d'un casque et d'un micro ou, plus souvent, grâce au chat intégré au jeu, le biais essentiel, spontané, de communication demeure corporel: on se fait comprendre à travers des gestes, des mouvements, des actions. La rencontre avec d'autres joueurs se fait toujours en confrontation avec leur corps virtuel ${ }^{27}$.

41 Le corps virtuel, en tant que support culturel oscille entre représentation de la naturalité (qui renvoie au corps humain et à sa spontanéité) et artificialité (qui se manifeste par la limitation des gestes contrôlés par le biais d'une manette ou d'un clavier $)^{28}$.

42 Le joueur dispose de nombreux réglages pour affiner la gestuelle corporelle de son avatar et préciser son registre expressif. Il a ainsi le choix parmi quelques expressions faciales (définies comme humeur du joueur, qui peut être "concentré », "énervé », « 
content », «blessé », «stressé », «fier », «normal» ou "boudeur »). Le manque d'expressivité faciale empêche cependant la reconnaissance des expressions psychologiques. Pour compenser cette perte de sens, le joueur doit s'investir davantage par le corps. Il y a donc un décalage entre un visage relativement figé et un corps en perpétuel mouvement. Ces mouvements corporels standardisés passent par différentes façons de marcher (le joueur peut alors adopter une « démarche de gangster ", une " démarche efféminée ", une "démarche snob», une "démarche de gros dur ", une " démarche dansante » ou une " démarche normale »). Le joueur peut aussi choisir parmi plusieurs types de saluts, dont bon nombre renvoie au registre outrancier voire grossier, et qui participe à la mise en scène parodique et satirique propre à GTA Online.

De façon générale, le corps du joueur renvoie ici, tout comme un corps de théâtre, aux dépendances du corps face aux codes sociaux et culturels, sa palette d'expressions corporelles renvoyant aux "conditionnements posturaux" et à une certaine " aliénation gestuelle » standardisée ${ }^{29}$. Le corps dans GTA Online joue avec les limites de ce que la culture définit comme étant un corps contrôlé et un corps non maîtrisé (gestes lents, rapides, hystérisés).

Le corps du joueur est ainsi un corps déformable, un corps dénué de douleur, sans cesse soumis aux blessures et aux meurtrissures. La mort est toujours transitoire et réversible, elle n'a rien de définitif. La représentation du corps qui se meurt s'inscrit la plupart du temps dans un registre comique, comme c'est le cas lorsque le joueur tombe d'une certaine hauteur ${ }^{30}$ : la scène de la chute n'est alors pas sans rappeler celle qui prévaut dans l'univers des cartoons hollywoodiens, en particulier ceux produits par Tex Avery.

Même s'il a un rapport corporel et dynamique avec son environnement immédiat, le joueur, qui se perçoit lui-même à la troisième personne, est cependant dirigé à distance : il est ce pantin dont parle Diderot lorsqu'il évoque le jeu de l'acteur dans le Paradoxe sur le comédien ou la sur-marionnette fantasmée et théorisée en 1911 par le metteur en scène Gordon Craig ${ }^{31}$ qui prétendait au contrôle absolu sur le corps de l'acteur, qui devenait machine ${ }^{32}$.

46 En raison de la prévalence de la vue à la troisième personne ${ }^{33}$, le corps avatarial est autant une marionnette qu'un comédien. Une marionnette manipulée non plus par une croix d'attelle, mais par une manette. Le corps de l'avatar oscille ainsi entre corps naturel et corps-marionnette. Au-delà du rapport visuel, gestuel et corporel, cette continuité se prolonge également sur le plan du récit : le théâtre de Guignol se situe lui aussi, tout comme GTA Online, dans un rapport de renversement vis-à-vis de l'autorité. C'est le jeu des gendarmes et des voleurs, et Guignol, encouragé par le public, peut frapper le gendarme. C'est le rire par la bastonnade ${ }^{34}$. Mais qu'il soit corps-marionnette ou corps de comédien (les deux versants ne sont pas incompatibles), le corps du joueur est surtout un corps costumé et souvent masqué.

Tout comme le costume de théâtre, le costume est ici signe d'identification du personnage, les variations de tenues n'offrant que très peu de différences du point de vue fonctionnel. Le costume peut ainsi confiner au déguisement par effet de grossissement, de simplification et de lisibilité. L'hypertrophie esthétique du costume renvoie aux excès et à l'exagération du personnage joué par le joueur ${ }^{35}$. Le costume peut ainsi constituer l'expression d'un style de jeu (par exemple jouer en costume militarisé), d'une distance ironique (jouer en peignoir), d'un jeu de miroir (se représenter soi-même). Le costume de l'avatar est un corps socialisé qui donne parfois 
des indices plus ou moins codés sur le sexe, parfois l'âge, plus rarement (voire jamais) sur le métier ou l'appartenance sociale du joueur. Cependant, la fonction signalétique $\mathrm{du}$ costume prend appui sur des codes culturels qui fonctionnent sur un double niveau déjà évoqué : à l'intérieur du système de la mise en scène ${ }^{36}$ et à l'extérieur du système de la mise en scène ${ }^{37}$.

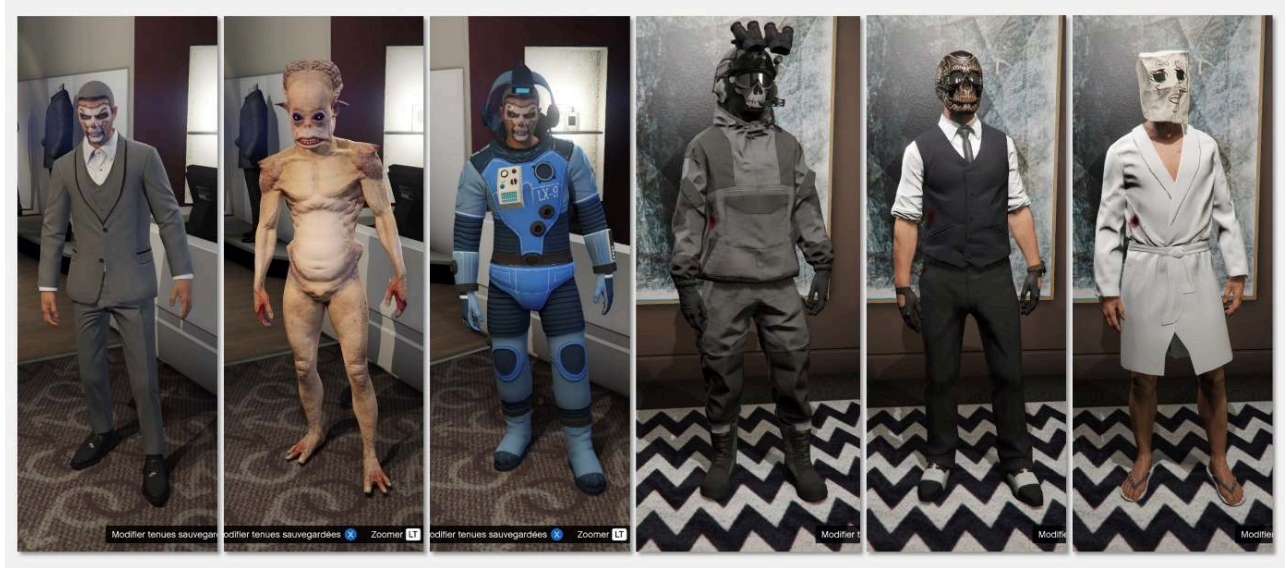

Image 1, différents costumes et déguisements mis à disposition dans GTA Online, Rockstar Games, Tous Droits Réservés

L'usage de masques renvoie quant à lui à des pratiques théâtrales aussi bien que militaires et criminelles, le plus souvent sur un registre caricatural. Les masques outranciers, la déformation des visages, renvoient aux fêtes masquées qui libèrent les identités et les interdits de sexe ou de classe, ainsi qu'au jeu masqué du théâtre latin, à la Commedia dell'arte ou théâtre japonais. Certains masques que l'on peut acquérir dans GTA Online font explicitement référence à ces traditions théâtrales comme aussi au théatre de mime ou aux masques des fêtes populaires. On trouve ainsi les masques d'Oni, en usage dans le théâtre japonais, les masques mexicains de la Fiesta de los muertos, des masques de style vénitien.

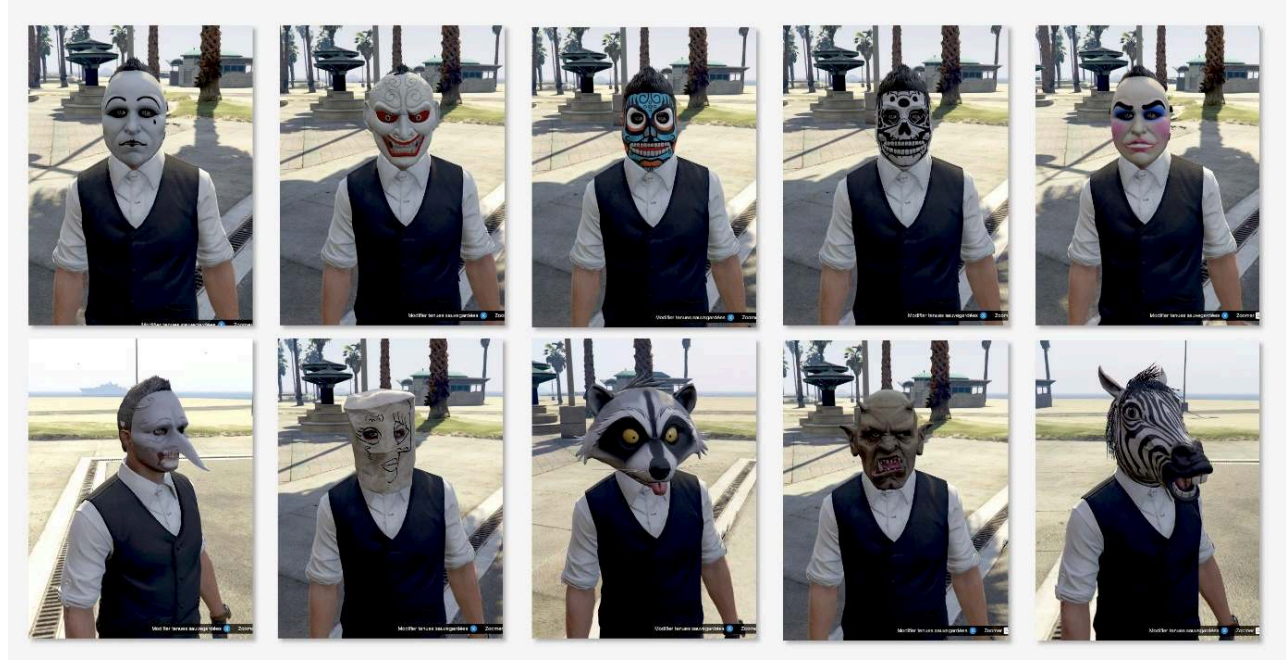

Image 2, masques dans GTA Online, Rockstar Games, Tous Droits Réservés 


\section{Une batmobile dans mon garage}

La projection d'un imaginaire s'applique également aux divers objets ${ }^{38}$, à savoir les armes et les véhicules, que GTA Online met à disposition des joueurs. La dimension expressive des objets se double ainsi de considérations fonctionnelles, puisque, contrairement aux accessoires vestimentaires, toutes les armes et tous les véhicules ne se valent pas du point de vue de leur efficacité. Cette dimension pratique, alimente tout une culture locale qui permet au joueur d'identifier l'arme la plus efficace dans une situation donnée, la voiture la plus rapide ou le véhicule au blindage le plus résistant.

Les nombreuses armes font partie des accessoires iconiques de GTA Online. Il en existe de tout type, du pistolet de combat au lance-missile, en passant par le fusil à canon scié et le fusil de précision lourd. De même pour les véhicules, à savoir les avions, hélicoptères, bateaux et voitures, dont près de 600 modèles sont en vente dans le jeu à ce jour. L'automobile occupe cependant une place centrale dans la franchise GTA : c'est le vol qualifié de voiture qui donne le nom à toute la franchise ${ }^{39}$ et la voiture constitue un objet de désir prédominant.

Les courses de voitures, la customisation de véhicules et le goût pour un certain type de modèles sont des facteurs importants de socialisation à l'intérieur du mode libre, dans la mesure où des rencontres sont organisées pour exposer les véhicules ou que des groupes sont créés en lien avec ces thèmes. Les véhicules qui se trouvent dans le jeu font tous référence à des véhicules existant dans la réalité, ils sont plus ou moins facilement identifiables malgré les subtiles nuances dans les formes qui permettent à l'éditeur Rockstar de contourner les règles de la propriété intellectuelle. Certains véhicules renvoient directement à des véhicules iconiques issus de la culture cinématographique ou de la culture pop ${ }^{40}$.

52 À titre d'exemple, la Ruiner renvoie explicitement à la voiture de la série télévisée Knight Rider, la Deluxo à la DeLorean de Retour vers le futur ${ }^{41}$, la Vigilante à la Batmobile du film Batman ${ }^{42}$ de Tim Burton, la Stromberg (qui peut se transformer en sous-marin) renvoie à la Lotus Esprit, elle aussi submersible, tirée du film L'Espion qui m'aimait ${ }^{43}$ sorti en 1977 et dans lequel l'adversaire de James Bond s'appelle, précisément, Karl Stromberg. Et pour finir, la Scramjet renvoie aux véhicules du manga Speed Racer créé par Tatsuo Yoshida en 1966.

Ces réappropriations culturelles misent bien entendu sur la convoitise des joueurs, qui dépenseront l'argent qu'ils ont gagné dans le jeu pour acquérir un véhicule emblématique de la culture pop. Mais il résulte de cette offre un syncrétisme culturel ironique qui rend possible le fait que, dans les rues de Los Santos, une Batmobile croise une DeLorean de Retour vers le Futur. Tous ces véhicules sont des objets porteurs de récits et, à ce titre, ils sont perçus comme étant iconiques et hautement désirables. Ce statut particulier se voit renforcé par un système publicitaire dont la rhétorique porte moins sur les qualités du véhicule que sur la place que celui-ci occupe à l'intérieur du renversement des valeurs qui s'opère dans GTA Online. Les descriptions que l'on trouve sur les sites de vente des véhicules cherchent à promouvoir des valeurs de consommation exactement opposées à nos publicités (dont elles soulignent ainsi

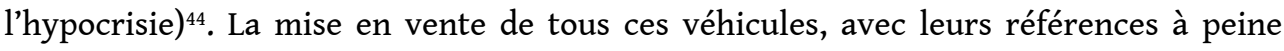
masquées, alimente chez les joueurs leur propension à l'accumulation et à la collection et fait fonctionner l'économie du jeu. Il en résulte un hyperconsumérisme, qui pousse à 
une accumulation presque sans fin (des voitures inédites sont encore mises en vente, et cela sept ans après la sortie du jeu).

\section{L'espace scénique}

trafic automobile n'obéit pas aux règles ordinaires de la circulation routière. Bien sûr, cela tient à ma vitesse excessive et à ma coupable négligence à l'heure de respecter les feux de signalisation et le sens de la circulation. Mais on dirait bien qu'il y a quelque chose de plus : certains véhicules cherchent délibérément à entrer en collision, ils se décalent au dernier moment, ils ne respectent aucune priorité et s'avancent pour me couper la route. Au fil du temps, le constat paraît évident : dans GTA Online, le trafic cherche à provoquer des accidents. Les stratégies sont nombreuses pour arriver à cette fin et le résultat est toujours le même : la carrosserie de mon véhicule ne reste jamais intacte très longtemps.

Si les accidents sont (par définition) la plupart du temps imprévus, il est possible de progressivement mettre en place des stratégies afin de provoquer des accrochages plus ou moins spectaculaires avec d'autres véhicules. Le spectre dans lequel s'inscrit l'accident, de l'imprévu au délibéré, est enregistré par un certain nombre d'exemples illustrés par des joueurs.

On trouve ainsi une discussion sur le forum Reddit dont le titre "This looks like a movie scene $»^{49}$ résume parfaitement la lecture qui en est faite, où l'accident imprévu renvoie à la cascade automobile, si caractéristique du cinéma d'action. À l'autre extrême du spectre, la chaîne youtube $\mathrm{EGCM}^{50}$ propose une playlist complète ${ }^{51}$ de séquences qui présentent des accidents provoqués, montrés au ralenti, dont la forme renvoie elle aussi au cinéma d'action. On devine que cette esthétique emphatique de

Cahiers de Narratologie, 38 | 2020 
l'accident routier exige une préparation et un contrôle soigneux des conditions de sa survenue. Elle n'est rendue possible que par la disposition inscrite dans le jeu et qui cherche à détruire la voiture du joueur. Le rapport fétichiste que le joueur entretient avec son automobile, en tant qu'objet culturel, et que le jeu n'a cessé d'encourager, vient exacerber les sentiments de frustration et de plaisir coupable qui sont ici en jeu.

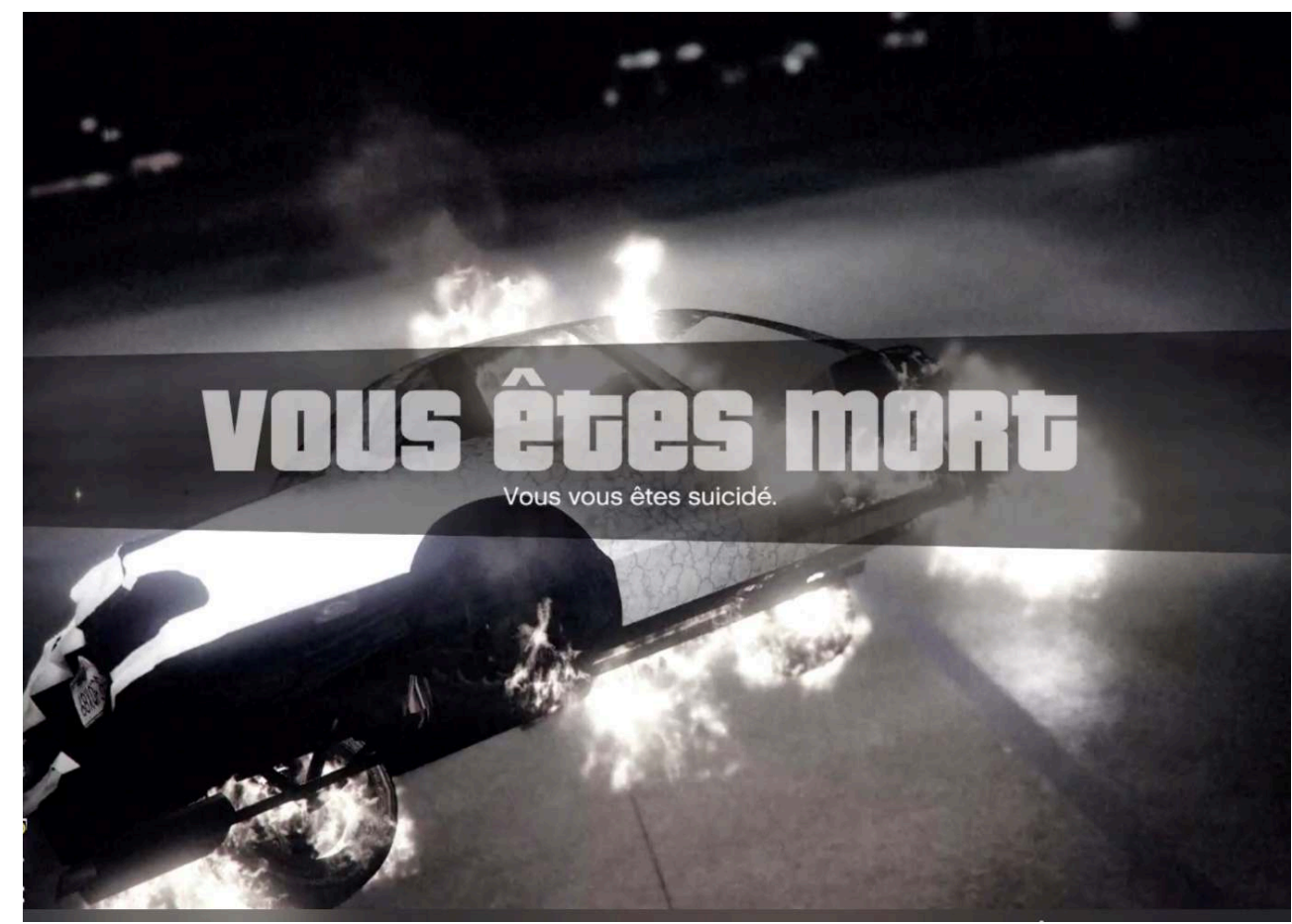

Image 3, la mort du joueur suite à un accident de voiture, Rockstar Games, Tous Droits Réservés

Mais c'est sur la route, en tant que lieu parabolique, que l'accident a lieu. La route, ses lacets, son trafic hostile et l'automobile customisée du joueur permettent de jouer et de rejouer la scène de l'accident de voiture. Les éléments visuels qui nourrissent le récit que le joueur peut faire de l'accident dont il est la victime renvoie à une esthétique de l'épave automobile qui peut faire penser à l'œuvre James Ballard, en particulier à son exposition Crashed Cars de 1970, qui a inspiré le roman Crash ! : l'esthétique de l'épave est ici prédominante, avec une sexualisation de l'accident de la route et une sublimation ironique de la voiture comme produit commercial industriel par excellence. Dans un texte de 1969, intitulé La Foire aux atrocités et qui précède l'exposition Crashed Cars, Ballard insistait déjà sur ce caractère iconique de la voiture :

Le rôle ambigu de l'accident de voiture ne requiert aucune élaboration - à

l'exception de notre propre mort, il est probablement l'événement le plus dramatique de notre existence, et dans de nombreux cas les deux coïncident. Audelà du fait que, généralement, nous sommes propriétaires du véhicule impliqué dans l'accident ou que nous sommes au volant, l'accident d'automobile diffère de tous les autres désastres en ce sens qu'il implique le produit commercial le plus puissamment publicisé de ce siècle, une entité iconique qui combine les éléments de vitesse, de puissance, de rêve et de liberté sous une forme hautement stylisée qui désamorce toutes les craintes que nous pouvons éprouver au sujet des dangers inhérents à ces violentes et instables machines ${ }^{52}$.

60 Cet exemple montre à quel point l'événement imprévu, qui prend le joueur au dépourvu et qui exige une réponse improvisée, tout comme les élaborations successives plus structurées qu'il peut mettre en œuvre pour systématiser la survenue de 
l'événement, s'inscrit dans une histoire culturelle précise, en l'occurrence celle de l'accident de voiture et de la critique de la société consumériste.

En puisant dans mon répertoire individuel, j'ai customisé ma voiture personnelle selon un système de représentations qui revoie à l'automobile en tant qu'objet culturel, mais aussi à ma propre expérience. J'ai joué et rejoué la scène de l'accident en exploitant la disposition du jeu à vouloir détruire ma voiture et à remettre en question le lien sentimental qui me rattache à elle. J'ai aussi usé des capacités du décor à créer les conditions propices d'une esthétisation cinématographique de l'accident. L'exercice du crash et ses réitérations peut bien se pratiquer en solitaire, il n'en demeure pas moins qu'il fait entrer en jeu des schémas d'action qui font appel à aux représentations collectives de la voiture et de l'accident de voiture, que celui-ci survienne de façon imprévue ou qu'il soit provoqué.

62 Et le récit de l'accident, qui vient finalement alimenter les archives collectives, proclame à quel point la scène filmée ou racontée fait penser à une scène de cinéma.

\section{L'attaque de la base militaire}

Dans une vidéo de 17 minutes, intitulée "On attaque la base militaire avec les abonnés $»^{53}$ et mise en ligne le 10 décembre 2013, le youtubeur MrQuoty procède à l'attaque de la base militaire dans GTA Online accompagné de 15 joueurs. Il s'agit de la première d'une série de sept vidéos d'une même série ${ }^{54}$.

Les règles, énoncées au début de la séquence, sont simples : les joueurs se réunissent dans une session du mode libre de GTA Online et, ensemble, ils doivent s'introduire dans la base militaire (qui se trouve au nord-ouest de l'île de San Andreas) et tenir le plus longtemps possible, face aux soldats qui demeurent en général pacifiques si personne ne s'introduit dans la base et si personne ne les attaque. Deux règles simples sont explicitement énoncées au début de la séquence: tenir au maximum en se déplaçant à sa guise dans la base et provoquer le plus de destruction possible à l'intérieur de celle-ci (peu importe les moyens).

Mais l'attaque menée par les joueurs est vouée à l'échec, les soldats éliminés sont remplacés au fur et à mesure par d'autres soldats qui apparaissent dans un flux continu et sans fin. Le seul objectif est de tenir et, lorsqu'un joueur est tué, il peut revenir dans la base militaire. Il n'y a pas de conditions de victoire ni de marqueurs de meilleure performance possible. L'attaque se déroule donc dans le plus grand désordre, chaque joueur multipliant les initiatives avec témérité et audace, les uns pour tenir le toit du hangar, les autres pour s'emparer d'un avion de chasse ou d'un tank.

Dans le cadre de cette séquence de jeu, les joueurs ne sont pas tout à fait dans le jeu de rôle ni dans le jeu orienté vers un but définitif à atteindre. Il s'agit d'une improvisation qui, en tant que pratique culturelle, autorise une liberté d'action dans un cadre donné. L'improvisation concerne ici l'action individuelle ou collective qui peut être orientée non pas vers un objectif précis, mais vers une situation de jeu dont la mise en œuvre ne répond pas à une stratégie mise en place à l'avance de façon explicite, seul le cadre est défini. Le joueur se laisse porter par un style de jeu et par les accessoires immédiatement à disposition. Il fait communauté par l'action qu'il partage avec d'autres joueurs. 
67 Dans le cadre prédéfini de ces improvisations, l'événement «attaque de la base militaire » est le résultat de l'interaction entre un répertoire individuel qui s'attache aux caractéristiques de l'avatar et aux accessoires qui l'accompagnent, mais aussi en tant que connaissance personnelle du jeu, de ses propriétés précises ${ }^{55}$ et un répertoire de travail en tant que répertoire collectif des actions réalisables dans la base militaire pour créer le plus grand désordre possible, coordination immédiate et spontanée qui fait que les joueurs finissent la plupart par se retrouver sur le toit du hangar, lieu le plus indiqué pour assurer les meilleures conditions de survie aux joueurs et les meilleures conditions pour détruire les objectifs qui se présentent.

Ces deux répertoires incluent une connaissance pratique des interactions avec les personnages non jouables, puisque, dans les situations décrites, leur rôle n'est pas limité à celui d'être de simples figurants au comportement prédéfini, mais plutôt des partenaires de jeu à part entière dans la production de l'événement. Il faut donc souligner le statut hybride des PNJ, qui font à la fois partie du décor, ${ }^{56}$ mais qui, dans le cas par exemple des policiers, des militaires et des membres de gang, endossent un rôle très caractérisé, un rôle d'opposant dont les références et le comportement agressif très défini en font de véritables partenaires de jeu.

69 À l'heure de considérer l'attaque de la base militaire sous l'angle du spectacle vivant, les conditions de jeu définies pourraient se rapprocher des directives données aux acteurs de la pièce The Brig, écrite par Kenneth $\mathrm{H}$. Brown. Cette pièce jouée pour la première fois par la troupe du Living Theater en 1963 à New York (mise en scène par Julian Beck et Judith Malina) présente des scènes de vie quotidienne dans un pénitencier des Marines. L'action de la pièce est déterminée par la structure du décor et par le véritable règlement disciplinaire d'une prison militaire, qui décrit les routines imposées aux détenus. Les comédiens, dont le nombre varie entre 10 et 30 en fonction des représentations, jouent les gardiens et les prisonniers. Ils s'en tiennent au décor pénitentiaire et au règlement pour improviser les différentes séquences, les prisonniers ayant l'interdiction de parler entre eux et les comédiens qui jouent les gardiens ayant une marge de manœuvre pour appliquer le règlement ${ }^{57}$.

70 Le rapprochement, qui mériterait un approfondissement, s'appuie sur le fait que, dans les deux cas, un décor est défini comme lieu unique du jeu et un système de règles est énoncé. Dans le cas de l'attaque de la base militaire, une fois le cadre spatial défini (la base militaire devient alors le décor à jouer), les actions individuelles improvisées peuvent se déployer en obéissant à deux règles simples, détruire tout ce qui se présente et tenir au maximum à l'intérieur de la base, tout en sachant que c'est une opération sans objectif définitif, dans la mesure où l'on ne peut que tenir le plus longtemps possible et mourir, sans jamais venir à bout des vagues infinies de soldats.

\section{Raconter l'imprévu}

71 Les quelques réflexions esquissées à partir de l'attaque de la base militaire et des accidents de voiture permettent de percevoir la richesse et la complexité des dynamiques narratives qui sont à l'œuvre dans les moments de creux, lorsqu'une automobile croise ma route et me percute, ou lorsque je suis pleinement engagé dans une activité improvisée sans autre but que la recherche de l'amusement. Sur fond de décor parabolique, les représentations portées par le corps costumé et par les accessoires et les véhicules empruntent leurs contours à plusieurs sources : elles font 
de GTA Online, en tant que monde ouvert multijoueur, un spectacle vivant hybride et éclectique, où les récits se superposent. Saturés de renvois multiples et de "reflets de systèmes d'idées " qui se mêlent à la culture locale du jeu, en particulier aux implications pratiques qu'elle comporte, les répertoires individuels et collectifs déterminent aussi bien l'apparence du corps avatarial que le style de jeu déployé par le joueur. En jouant à GTA online, celui-ci est donc en mesure de recycler des fragments de récits et de réécrire un récit d'une manière radicalement impure, en mêlant les éléments iconographiques et narratifs venus d'univers diégétiques différents. Les représentations du corps, de la violence et des relations sociales qui sont ici en œuvre reposent donc sur un éclectisme culturel assumé. Celui-ci est au service d'un renversement des valeurs qui se rapproche des règles de la transgression carnavalesque. Au-delà d'un rire par bastonnade ou de jouer à bandit et police, on retrouve dans GTA Online les accents de la « désacralisation de l'ordre dominant et de ses institutions ${ }^{58}$ propres au carnaval traditionnel, qui est lui aussi un spectacle vivant marqué par une esthétique polysémique. Dénuées de toute finalité mesurable en termes de score ou de gains matériels, les phases de jeu décrites plus haut reposent aussi sur un principe de rire carnavalesque, basé sur la destruction de véhicules, les accidents spectaculaires et les morts violentes. La double référence aux expérimentations théâtrales et aux improvisations du Jazz permet de percevoir le joueur du mode libre comme un cousin éloigné du comédien et du musicien. L'interaction entre un répertoire individuel et un répertoire de travail comme moteur de l'agir improvisé lui donne un petit air de famille.

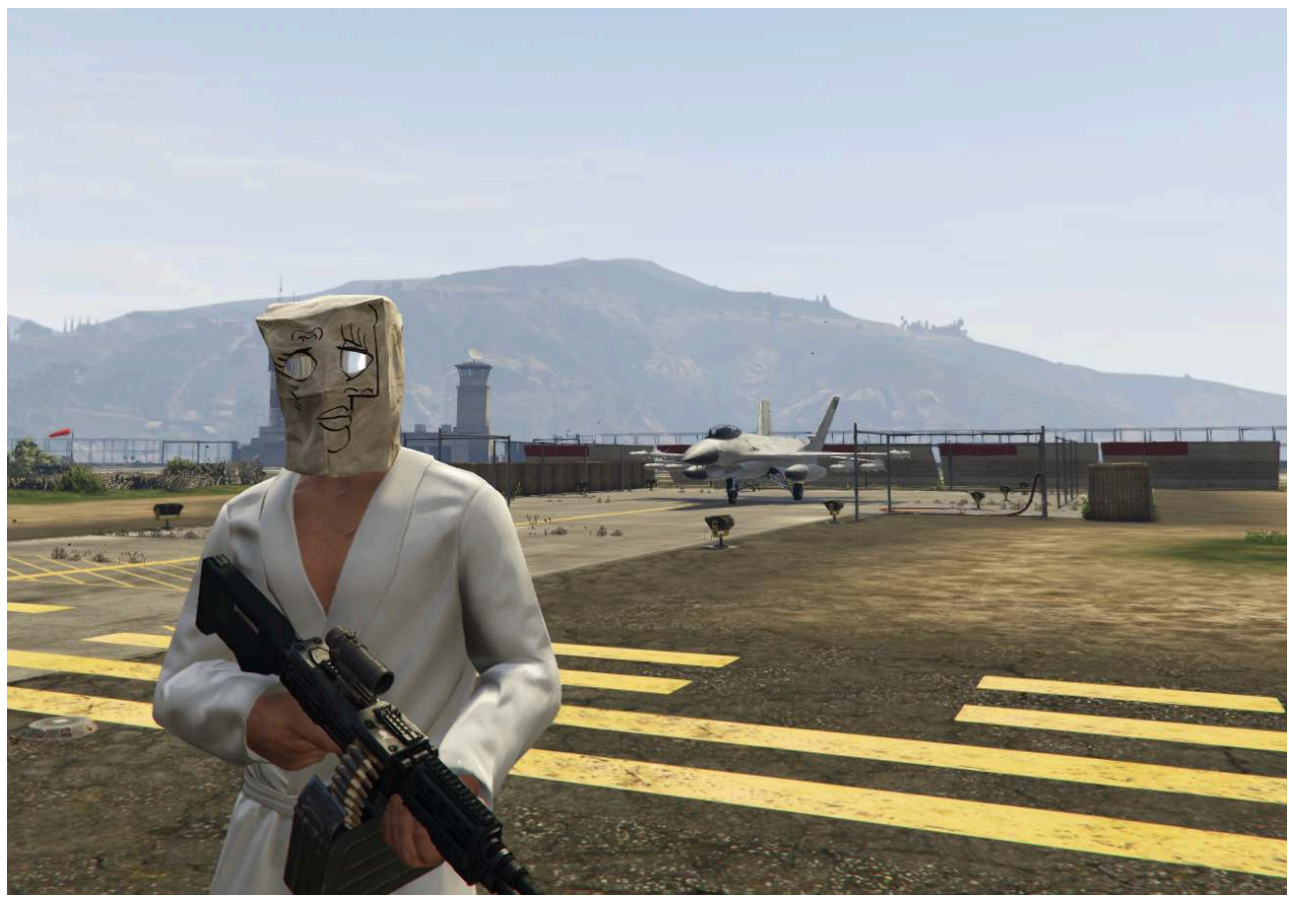

Image 4, peignoir et masque cubiste, Rockstar Games, Tous Droits Réservés

Si le fait de pénétrer dans la base militaire de San Andreas en peignoir et en portant un masque fait d'un sac de papier comportant un dessin cubiste, à bord d'une Batmobile, est de l'ordre d'une réécriture culturelle décomplexée et impure, qui met en action les citations prêtes à l'usage disposées dans le jeu, et qui s'apparente à un carnaval guignolesque assumé, il n'en reste pas moins qu'à un moment ou un autre, il va bien falloir raconter ce qui s'est passé dans l'enceinte de la base militaire. En effet, une fois 
le jeu terminé, il arrive au joueur de raconter la combinaison inédite et improvisée des actions, de fixer et de résumer ce qui vient d'arriver en une narration plus un moins cohérente. L'horizon de cette contribution est donc aussi d'évoquer la question " comment on raconte le jeu vidéo ? $\|^{59}$. Autrement dit, comment raconte-t-on aprèscoup les situations de jeu vécues et jouées.

La difficulté consiste à documenter cette étape, tant la plus large majorité des récits produits trouvent leur expression dans la sphère privée et n'est que très rarement conservée sur des supports accessibles. Ou alors, lorsque c'est le cas, ces récits sont souvent retravaillés et améliorés (en particulier quand ils font l'objet d'un montage vidéo qui élimine les redondances de l'action ou les moments creux), ils perdent de leur caractère «bricolé » et spontané. On touche ici à la question des pratiques ordinaires du jeu et à la conservation de ces «microrécits» qui ne sont que rarement préservés. Tout au plus reste-t-il quelques captures d'écran, quelques discussions sur le forum Reddit et des vidéos sur différents supports ${ }^{60}$. L'oralité joue ici un rôle prédominant, dans la mesure où la mémoire et la transmission des événements imprévus ou organisés passent la plupart du temps par un récit oral à la première personne. Mais ces micros-récits réarrangés ne sont pas anodins. L'intérêt que l'on peut porter à ces témoignages des pratiques ordinaires n'est pas éloigné de l'intérêt manifesté par l'historien et anthropologue Daniel Fabre envers ce qu'il appelle "les écritures ordinaires ", ces écritures du banal et du quotidien (liste de choses à faire, légendes de photos, cartes postales), qui n'appartiennent pas à ces « écrits que distingue la volonté de faire œuvre $"^{61}$, mais qui témoignent de pratiques culturelles et de compétences qui sont au centre de la vie des individus.

\section{BIBLIOGRAPHY}

ASLAN, Odette, AUBERT, Christiane, BOURBONNAUD, Jean-Luc, JACQUOT, Jean et OUAKNINE, Serge, 1970. Les voies de la création théâtrale. Paris : CNRS éditions.

BALLARD, James G., 2003. La Foire aux atrocités. Auch : Tristram.

BARNABÉ, Fanny, 2018. Narration et jeu vidéo : Pour une exploration des univers fictionnels. Liège : Presses universitaires de Liège. Disponible à l'adresse : http://books.openedition.org/ pulg/2613 [dernière consultation le 18 juillet 2020].

BARNABÉ, Fanny et DELBOUILLE, Julie, 2018. Aux frontières de la fiction : l'avatar comme opérateur de réflexivité. In : Sciences du jeu. 2018. Vol. 9. Disponible à l'adresse : http:// journals.openedition.org/sdj/958 [dernière consultation le 18 juillet 2020].

BECKER, Howard S. et FAULKNER, Robert R., 2011. « Qu'est-ce qu'on joue, maintenant? », Le répertoire de jazz en action. Paris : La Découverte.

BECKER, Howard S., FAULKNER, Robert R. et KIRSHENBLATT-GIMBLETT, Barbara (éd.), 2006. Art from Start to Finish: Jazz, Painting, Writing, and Other Improvisations. Chicago : University of Chicago Press. 
BERLINER, Paul F., 1994. Thinking in Jazz. The infinite art of improvisation. Chicago : University of Chicago Press.

BOUTET, Manuel, 2012. Jouer aux jeux vidéo avec style. Pour une ethnographie des sociabilités vidéoludiques. In : Réseaux. 2012. Vol. 3-4, p. 207-234.

BOUTET, Manuel, COLÓN DE CARVAJAL, Isabel, MINASSIAN, Hovig Ter et TRICLOT, Mathieu. Audelà du virtuel: interactions sociales et spatiales dans et autour d'un univers vidéoludique. In : Médiation et information. $n^{\circ} 37$, p. 103-116.

CAÏRA, Olivier, 2014. Jeux vidéo et jeux d'interaction en face-à-face : vers un modèle unifié d'écologie de l'intrigue. In : Cahiers de Narratologie. 2014. Vol. 27. Disponible à l'adresse : http:// narratologie.revues.org/7058 [dernière consultation le 18 juillet 2020].

CAÏRA, Olivier, 2016. Théorie de la fiction et esthétique des jeux. In : Sciences du jeu. 2016. Vol. 6. Disponible à l'adresse : http://sdj.revues.org/671 [dernière consultation le 18 juillet 2020].

CAÏRA, Olivier, 2018. Les dimensions multiples de l'engagement ludique. In : Sciences du jeu. 2018. Vol. 10. Disponible à l'adresse : http://journals.openedition.org/sdj/1149 [dernière consultation le 18 juillet 2020].

CAÏRA, Olivier, 2019. Qu'allez-vous faire de Roméo ? Des « sentiers qui bifurquent » aux nouveaux outils d'écriture interactive. In : Contextes. 2019. n²2, p. 1-13.

CAYATTE, Rémi, 2018. Temps de la chose-racontée et temps du récit vidéoludique : comment le jeu vidéo raconte? In : Sciences du jeu. 2018. Vol. 9. Disponible à l'adresse : http:// journals.openedition.org/sdj/936 [dernière consultation le 18 juillet 2020].

CRAIG, Edward Gordon, 1999. De l'art du théâtre. Belfort : Circé.

FABRE, Daniel (éd.), 1993. Ecritures ordinaires. Paris : P.O.L.

GOLDBERG, Roselee, 2001. La Performance, du futurisme à nos jours. Paris : Thames \& Hudson.

GUIDICELLI, Carole (éd.), 2013. Surmarionnettes et mannequins. Crag, Kantor et leurs héritages contemporains. Lavérune : L’Entretemps - Institut International de la Marionnette.

JOLIVEAU, Thierry, 2012. Les Lieux Réels Dans Le Jeu Vidéo. Contribution à une approche géographique des espaces vidéoludiques. In : MINASSIAN, Hovig Ter, RUFAT, Samuel et TRICLOT, Mathieu (éd.), Espaces et temps des jeux vidéo. Paris : Questions théoriques. p. 148-181.

KRICHANE, Selim, 2014. L'intrigue en trois dimensions Les récits vidéoludiques à l'ère des polygones, de la « caméra virtuelle » et du CD-ROM. In : Cahiers de Narratologie. 2014. Vol. 27. Disponible à l'adresse : http://narratologie.revues.org/7014 [dernière consultation le 18 juillet 2020].

KRICHANE, Selim, 2018. La Caméra imaginaire. Jeux vidéo et modes de visualisation. ChêneBourg : Georg éditeur.

LAHIRE, Bernard, 2006. La culture des individus. Dissonances culturelles et distinction de soi. Paris : La Découverte.

LE BOEUF, Patrick, 2013. La Surmarionnette, source de féconds malentendus. In : GUIDICELLI, Carole (éd.), Surmarionettes et Mannequins: Craig, Kantor et leurs héritages. Lavérune : L'Entretemps - Institut International de la Marionnette. p. 45-53.

LEMONNIER, Pierre, 2013. De l'immatériel dans le matériel... et réciproquement ! Techniques et communication non verbale. In : Journal de la Société des Océanistes. 2013. n 136-137, p. 15-26. 
LEWIS, George E. et PIEKUT, Benjamin (éd.), 2016. The Oxford Handbook of Critical Improvisation Studies. New York : Oxford University Press.

LIGNEREUX, Aurélien, 2003. Rosser le gendarme dans les spectacles de marionnettes au XIXe siècle : une école de rébellion? In : Sociétés \& Représentations. 2003. Vol. 2, n 16, p. 95-113.

LUCAS, Jean-François, 2018. Les figures de l'habitant dans les mondes virtuels. In : Sciences du jeu. 2018. Vol. 10. Disponible à l'adresse : http://journals.openedition.org/sdj/1353 [dernière consultation le 18 juillet 2020].

MARTI, Marc, 2014. La narrativité vidéoludique : une question narratologique. In : Cahiers de Narratologie. 2014. Vol. 27, p. 1-15. Disponible à l'adresse : http:// journals.openedition.org/ narratologie/7009 [dernière consultation le 18 juillet 2020].

MAUCO, Olivier, 2013. GTA IV, l'envers du rêve américain. Paris : Questions théoriques.

MAUFFRET, Blodwenn, 2019. La matière au sein du carnaval : un constituant esthétique polysémique. In : Agôn. 2019. n 8, p. 1-22.

PAVIS, Patrice, 2019. Dictionnaire du théâtre. Paris : Armand Colin.

PICARD, Martin, 2016. Les enjeux esthétiques du jeu vidéo : entre art, stylistique et interactivité. In : Sciences du jeu. 2016. Vol. 6. Disponible à l'adresse : http://sdj.revues.org/712 [dernière consultation le 18 juillet 2020].

PICHLMAIR, Martin, 2008. Grand Theft Auto IV Considered as an Atrocity Exhibition. In : Eludamos. Journal for Computer Game Culture. 2008. Vol. 2, nº 2, p. 293-296.

SALEN TEKINBAş, Katie et ZIMMERMAN, Eric, 2003. Rules of Play. Game Design Fundamentals. Cambridge (Massachussetts) : MIT Press.

TRÉPANIER-JOBIN, Gabrielle et COUTURIER, Alexane, 2018. L'immersion fictionnelle au-delà de la narrativité. In : Sciences du jeu. 2018. Vol. 9. Disponible à l'adresse : http:// journals.openedition.org/sdj/950 [dernière consultation le 18 juillet 2020].

VENDEVILLE, Stéphanette, 2007. Le Living Theatre: de la toile à la scène, 1945-1985. Paris : L'Harmattan.

WALSH, Richard, 2011. Emergent Narrative in Interactive Media. In : Narrative. 2011. Vol. 19, nº 1, p. 72-85.

ZEILINGER, Martin, 2018. Survival Interventions in GTA: On the Limits of Performance in Virtual Environments. In : Video Game Art Reader. 2018. Vol. 2, n 1, p. 15-27.

\section{NOTES}

1. Voir WALSH, Richard, 2011. Emergent Narrative in Interactive Media. In : Narrative. 2011. Vol. 19, n 1, p. 72-85 et SALEN TEKINBAş, Katie et ZIMMERMAN, Eric, 2003. Rules of Play. Game Design Fundamentals. Cambridge (Massachussetts): MIT Press, en particulier p. 383. Voir aussi MARTI, Marc, 2014. La narrativité vidéoludique: une question narratologique. In : Cahiers de Narratologie. 2014. Vol. 27, p. 1-15, en particulier p. 8-9.

2. En fonction de la plateforme: 16 joueurs pour la version PS3 et Xbox 360, 30 personnes pour la version PS4, Xbox one et PC. 
3. Au sujet du passage de la vue aérienne à la «vue caméra » à la fin des années 90 , voir KRICHANE, Selim, 2018. La Caméra imaginaire. Jeux vidéo et modes de visualisation. ChêneBourg : Georg éditeur, p. 298.

4. On peut renvoyer ici au livre MAUCO, Olivier, 2013. GTA IV, l'envers du rêve américain, Paris : Questions théoriques, p. 10.

5. Malgré tout, ce séjour revêt d'un caractère provisoire qui semble suggérer que son ascension sociale peut s'avérer pour le moins éphémère.

6. A ce titre, il s'inscrit dans la continuité de la franchise GTA, voir MAUCO, Olivier, 2013. GTA IV, l'envers du rêve américain, p. 38.

7. Les liens entre l'oeuvre d'Ellroy et les productions de Rockstar sont nombreuses et connues: ainsi, le jeu L.A. Noir, édité par Rockstar en 2011, fait référence au L.A. Quartet d'Ellroy, c'est-à-dire quatre romans qui se déroulent dans le Los Angeles des années 1940-1950 : Le Dahlia Noir, Le Grand Nulle Part, L.A. Confidential et White Jazz.

8. Brian De Palma, 1983.

9. Michael Mann, 1995.

10. Quentin Tarantino, 1992.

11. Lethal Weapon 2, Richard Donner, 1989.

12. Harold Ramis, 1999.

13. D’après les indices parfois contradictoires disséminés à travers la map, on peut plus ou moins établir que GTA Online se déroule dans les années 2013-2017, mais cette datation tend à évoluer, voire à se brouiller, au fil de l'ajout de contenus additionnels.

14. A ce propos, voir aussi JOLIVEAU, Thierry, 2012. Les Lieux Réels Dans Le Jeu Vidéo. Contribution à une approche géographique des espaces vidéoludiques. In : MINASSIAN, Hovig Ter, RUFAT, Samuel et TRICLOT, Mathieu (éd.), Espaces et temps des jeux vidéo. Paris : Questions théoriques. p. 148-181.

15. Lester organise ainsi les braquages du mode online, Martin Madrazo, Lamar Davis et Trevor proposent des missions.

16. Plus d'une trentaine de DLC à ce jour. Des DLC supplémentaires sont prévus pour la deuxième partie de l'année 2020 et pour 2021. Le mode histoire n'a quant à lui connu aucun nouveau contenu.

17. A l'instar de la collecte de ressources pour les différentes affaires commerciales, des ventes de voitures, des ventes d'armes, ainsi que certaines missions de préparation aux braquages.

18. A propos du rôle de l'argent comme indicateur de score, voir aussi MAUCO, Olivier, 2013. GTA IV, l'envers du rêve américain, p. 89.

19. Pour un exemple de trajets périlleux en taxi (activité connue sous le nom de Taxi challenge), voir https://youtu.be/etocoV7fLF0 [vidéo en ligne consultée le 18 juillet 2020]. D'autres exemples aurait pu être ajoutés, tel que le braquage d'épiceries avec, pour seul véhicule pour échapper à la police, un vélo BMX, voir https://youtu.be/_qqKJxl_1Dk et https://youtu.be/hbrqWUCh8EU [vidéo en ligne consultée le 18 juillet 2020]).

20. BECKER, Howard S. et FAULKNER, Robert R., 2011. "Qu'est-ce qu'on joue, maintenant? ", Le répertoire de jazz en action. Paris : La Découverte.

21. Op. cit., p. 47-49. Voir aussi LEWIS, George E. et PIEKUT, Benjamin (éd.), 2016. The Oxford Handbook of Critical Improvisation Studies. New York : Oxford University Press, p. 402, qui insiste également sur l'idée d'une liberté donnée, mais soumise à une structure de contrainte. 
22. LEMONNIER, Pierre, 2013. De l'immatériel dans le matériel... et réciproquement ! Techniques et communication non verbale. In : Journal de la Société des Océanistes. 2013. $\mathrm{n}^{\circ}$ 136-137, p. 16-17.

23. Ibid., p. 17.

24. Ibid., p. 15.

25. Ibid., p. 18.

26. L'avatar, à la fois outil et personnage, voir BARNABÉ, Fanny et DELBOUILLE, Julie, 2018. Aux frontières de la fiction : l'avatar comme opérateur de réflexivité. In : Sciences $d u \quad$ jeu [en ligne]. 2018. Vol. 9, \$32. Disponible à l'adresse: http:// journals.openedition.org/sdj/958. Voir aussi BARNABÉ, Fanny, 2018. Narration et jeu vidéo: Pour une exploration des univers fictionnels [en ligne]. Liège : Presses universitaires de Liège, p. 47-49. Disponible à l'adresse : http://books.openedition.org/pulg/2613.

27. L'idée d'une confrontation est à peine métaphorique dans le cas de GTA Online, puisque le duel PvP constitue parfois la forme que prend spontanément une première rencontre avec d'autres joueurs. Mais je laisse cet aspect de côté pour l'instant.

28. Voir également PAVIS, Patrice, 2019. Dictionnaire du théâtre. Paris : Armand Colin, article « corps », p. 118-120.

29. Ibid., p. 119.

30. Voir par exemple les séquences proposées par la chaîne youtube ProGamingStudioHD dans la vidéo intitulée "GTA 5 Funny/Crazy Jump Compilation», https://youtu.be/p9k837ThXvk [dernière consultation le 18 juillet 2020].

31. CRAIG, Edward Gordon, 1999. De l'art du théâtre. Belfort: Circé, en particulier p. 79-106, article « L'acteur et la sur-marionnette », publié à l'origine en 1908.

32. Voir LE BOEUF, Patrick, 2013. La Surmarionnette, source de féconds malentendus. In : GUIDICELLI, Carole (éd.), Surmarionettes et Mannequins: Craig, Kantor et leurs héritages. Lavérune : L'Entretemps - Institut International de la Marionnette. p. 45-53.

33. Même si la vue à la première personne a été implémentée, GTA est traditionnellement un jeu qui se joue à la troisième personne.

34. Je renvoie à l'article LIGNEREUX, Aurélien, 2003. Rosser le gendarme dans les spectacles de marionnettes au XIXe siècle: une école de rébellion? In : Sociétés \& Représentations. 2003. Vol. 2, $\mathrm{n}^{\circ} 16, \mathrm{p} .95-113$. Ce spectacle du rire par la bastonnade et par le renversement des valeurs était, dans la pratique, en partie improvisé.

35. PAVIS, Patrice, 2019. Dictionnaire du théâtre, article « costume», p. 121.

36. Par exemple, les t-shirts qui mentionnent des marques ou des lieux propres à GTA. Voir aussi PAVIS, Patrice, 2019. Dictionnaire du théâtre, article « costume », p. 121.

37. Comme référence à nos propres pratiques vestimentaires.

38. A propos de la notion d'objet dans le jeu vidéo, voir BARNABÉ, Fanny, 2018. Narration et jeu vidéo : Pour une exploration des univers fictionnels [en ligne]. Liège : Presses universitaires de Liège, p. 101-106. Disponible à l'adresse: http:// books.openedition.org/pulg/2613.

39. Voir MAUCO, Olivier, 2013. GTA IV, l'envers du rêve américain, p. 26-27.

40. Voir ibid., p. 40.

41. Back to the Future, Robert Zemeckis, 1985.

42. Tim Burton, 1989.

43. The Spy Who Loved Me, Lewis Gilbert, 1977. 
44. A propos du système publicitaire dans GTA IV, mais le même propos peut s'appliquer à GTA V et GTA Online, voir MAUCO, Olivier, 2013. GTA IV, l'envers du rêve américain, p. 39-45.

45. Voir PAVIS, Patrice, 2019. Dictionnaire du théâtre, article « décor », p. 132.

46. A propos du décor, voir aussi BARNABÉ, Fanny, 2018. Narration et jeu vidéo : Pour une exploration des univers fictionnels [en ligne]. Liège : Presses universitaires de Liège, p. 113. Disponible à l'adresse : http://books.openedition.org/pulg/2613.

47. PAVIS, Patrice, 2019. Dictionnaire du théâtre, p. 132.

48. Ibid., article " espace scénique », p. 202.

49. Forum Reddit: https://www.reddit.com/r/gtaonline/comments/b61eue/ this_looks_like_a_movie_scene/ [dernière consultation le 18 juillet 2020]. Dans le même ordre d'idées, toujours sur Reddit, voir aussi https://www.reddit.com/r/gtaonline/comments/7v5e0e/ unintentional_action_movie_scene/ [dernière consultation le 18 juillet 2020] et https:// www.reddit.com/r/gtaonline/comments/bf6f83/everyday_is_like_a_movie_in_this_game/ [dernière consultation le 18 juillet 2020].

50. Voir https://www.youtube.com/c/EmuGaming/ [dernière consultation le 18 juillet 2020].

51. Voir https://www.youtube.com/playlist?list=PLZnMSNDgQ1z4BtV_PXSTH6haD2eCauc0V [dernière consultation le 18 juillet 2020].

52. BALLARD, James G., 2003. La Foire aux atrocités. Auch : Tristram, p. 179. Les liens qui unissent GTA IV et La Foire aux atrocités ont été soulignés dans l'article PICHLMAIR, Martin, 2008. Grand Theft Auto IV Considered as an Atrocity Exhibition. In : Eludamos. Journal for Computer Game Culture. 2008. Vol. 2, n² 2, p. 293-296.

53. https://youtu.be/GxHh2vBVCh8 [dernière consultation le 18 juillet 2020].

54. Mise en ligne de décembre 2013 à mai 2016

55. Dans le cas de l'attaque de la base militaire, la connaissance du jeu permet au joueur de savoir où se trouvent les avions de chasse, les tanks, et comment s'en emparer.

56. La fonction décorative des piétons se résumant par exemple à améliorer l'impression de réalisme de l'ensemble d'une scène.

57. On évoquer d'autres pièces, qui jouent sur les mêmes rapports entre décor, instructions données aux comédiens, mise en scène et improvisation, dans le cadre notamment des explorations théâtrales du Living Theater menées de 1947 jusqu'au milieu des années 80 et qui interrogent les limites du genre dramatique. A ce sujet voir ASLAN, Odette, AUBERT, Christiane, BOURBONNAUD, Jean-Luc, JACQUOT, Jean et OUAKNINE, Serge, 1970. Les voies de la création théâtrale. Paris: CNRS éditions, en particulier, à propos de The Brig, p. 183-192; voir aussi VENDEVILLE, Stéphanette, 2007. Le Living Theatre: de la toile à la scène, 1945-1985. Paris : L'Harmattan.

58. MAUFFRET, Blodwenn, 2019. La matière au sein du carnaval: un constituant esthétique polysémique. In : Agôn. 2019. n 8, p. 7.

59. Pour reprendre les termes d'un article de CAYATTE, Rémi, 2018. Temps de la choseracontée et temps du récit vidéoludique : comment le jeu vidéo raconte ? In : Sciences du jeu [en ligne]. 2018. Vol. 9. Disponible à l'adresse : http://journals.openedition.org/sdj/ 936.

60. A cet égard, les recherches effectués dans les sites Archive of Our Own (https:// archiveofourown.org/), FanFiction (https://www.fanfiction.net/) ou encore Wattpad (https://www.wattpad.com/) ont mis en évidence des récits qui font appel à des personnages ou à certains éléments tirés de GTA V et, plus rarement, de GTA Online. Il existe également des récits directement liés à des serveurs multijoueurs de roleplay qui 
se déroulent dans l'univers de GTA V, voir par exemple https://www.wattpad.com/ 789695293-la-nuit-des-morts-fini-avant-propos [dernière consultation 22 septembre 2020]. Cependant, ces récits, qui tirent très librement parti des éléments narratifs issus de GTA V et de GTA Online, ne relèvent pas vraiment du récit documentaire qui rendrait compte d'une séquence de jeu improvisé ou d'activités qui explorent et exploitent les mécaniques ludiques propres à GTA Online et dont il a été ici question.

61. FABRE, Daniel (éd.), 1993. Ecritures ordinaires. Paris : P.O.L., p. 11 ; pour quelques exemples d'œuvres et de performances à vocation artistique basées sur GTA, voir ZEILINGER, Martin, 2018. Survival Interventions in GTA: On the Limits of Performance in Virtual Environments. In : Video Game Art Reader. Vol. 2, n 1, p. 15-27.

\section{ABSTRACTS}

This article offers some thoughts about the freeroam in GTA Online. The aim is to highlight the complexity of the playful and narrative dynamics at work in very particular game phases, i.e. the moments when the player is confronted with unforeseen events (which he will then try to organize and systematize) or when he is fully engaged in an improvised but organized activity, with no other goal than the search for fun. Clothing, weapons, vehicles, and set elements allow players to exploit, combine, and rewrite narrative patterns from film, television, or literary culture, following codes that may be similar to the improvisation practiced by jazz players or actors. These action patterns make GTA Online, as an open multiplayer world, a hybrid and eclectic living theater.

Cet article propose quelques réflexions concernant le mode libre de GTA Online. À partir de quelques exemples choisis, le but est de mettre en évidence la richesse et la complexité des dynamiques ludiques et narratives qui sont à l'œuvre dans des phases de jeu très particulières, à savoir les moments où le joueur est confronté à des événements imprévus (qu'il va chercher ensuite à organiser et systématiser) ou lorsqu'il est pleinement engagé dans une activité improvisée, mais organisée, sans autre but que la recherche de l'amusement. Les accessoires vestimentaires, les armes, les véhicules et les éléments du décor permettent aux joueurs d'exploiter, de combiner et de réécrire des schémas narratifs issus de la culture cinématographique, télévisuelle ou littéraire, en suivant des codes qui pourraient s'apparenter à la pratique de l'improvisation que pratiquent les joueurs de jazz ou les comédiens. Cette mise en action de la culture fait de GTA Online, en tant que monde ouvert multijoueur, un spectacle vivant hybride et éclectique.

\section{INDEX}

Mots-clés: narration, jeux vidéo, improvisation, GTA, GTA Online, spectacle vivant, théâtre, jazz Chronological index: 2013-2020

Geographical index: Etats-Unis, Californie, San Andreas, Los Santos

Keywords: storytelling, story, video games, improvisation, GTA, GTA Online, live performance, theatre, jazz 